\title{
Nanoskiving Core-Shell Nanowires: A New Fabrication Method for Nano-optics
}

\section{Citation}

Watson, Douglas C., Ramses V. Martinez, Yannik Fontana, Eleonora Russo-Averchi, Martin Heiss, Anna Fontcuberta i Morral, George M. Whitesides, and Marko Lon?ar. 2014. "Nanoskiving CoreShell Nanowires: A New Fabrication Method for Nano-Optics." Nano Lett. 14 (2) (February 12): 524-531. doi:10.1021/nl403552q.

\section{Published Version}

doi: $10.1021 / \mathrm{nl} 403552 \mathrm{q}$

\section{Permanent link}

http://nrs.harvard.edu/urn-3:HUL.InstRepos:25811029

\section{Terms of Use}

This article was downloaded from Harvard University's DASH repository, and is made available under the terms and conditions applicable to Open Access Policy Articles, as set forth at http:// nrs.harvard.edu/urn-3:HUL.InstRepos:dash.current.terms-of-use\#OAP

\section{Share Your Story}

The Harvard community has made this article openly available.

Please share how this access benefits you. Submit a story.

\section{Accessibility}




\section{Nanoskiving Core-shell Nanowires: A New}

\section{Fabrication Method for Nano-optics}

Douglas C. Watson ${ }^{1,2 \ddagger}$, Ramses V. Martinez ${ }^{3,4 \neq}$, Yannik Fontana ${ }^{2}$,Eleonora Russo-Averchi ${ }^{2}$, Martin Heiss $^{2}$, Anna Fontcuberta i Morral ${ }^{2}$, George M. Whitesides ${ }^{3,5,6 *}$, and Marko Lončar ${ }^{1 *}$

AUTHOR ADDRESS.

1. School of Engineering and Applied Sciences, Harvard University, 33 Oxford Street, Cambridge, MA 02138, USA.

2. Laboratory of Semiconductor Materials, Institute of Materials, Ecole Polytechnique Fédérale de Lausanne, CH-1015 Lausanne, Switzerland.

3. Department of Chemistry and Chemical Biology, Harvard University, 12 Oxford Street, Cambridge, MA 02138, USA.

4. Madrid Institute for Advanced Studies, IMDEA Nanoscience, Calle Faraday 9, Ciudad Universitaria de Cantoblanco, 28049 Madrid, Spain.

5. Wyss Institute for Biologically Inspired Engineering, Harvard University, 60 Oxford Street, Cambridge, MA 02138, USA.

6. Kavli Institute for Bionano Science \& Technology, Harvard University, 29 Oxford Street, Cambridge, MA 02138, USA.

$\neq$ These authors contributed equally to this work.

* Corresponding authors emails:

gwhitesides@gmwgroup.harvard.edu, loncar@seas.harvard.edu 


\begin{abstract}
This paper describes the fabrication of functional optical devices by sectioning quantum-dotin-nanowires systems with predefined lengths and orientations. This fabrication process requires only two steps: embedding the nanowires in epoxy, and using an ultramicrotome to section them across their axis ("nanoskiving"). This work demonstrates the combination of four capabilities: i) the control of the length of the nanowire sections at the nanometer scale; ii) the ability to process the nanowires after cutting using wet etching; iii) the possibility of modifying the geometry of the wire by varying the sectioning angle; and iv) the generation of as many as 120 consecutive slabs bearing nanowires which have uniform size and approximately reproducible lateral patterns, and which can subsequently be transferred to different substrates. The quantum dots inside the nanowires are functional and of a high optical quality after the sectioning process, and exhibit photoluminescent emission with wavelengths in the range of $650-710 \mathrm{~nm}$.
\end{abstract}

KEYWORDS. Optics, nanowire, quantum dot, nanoskiving, core-shell, ultramicrotome. 
Quantum-dot-in-nanowires systems (QD-in-NWs) present optical properties promising for quantum optics, energy harvesting, and nano-sensing, by coupling the emission of quantum dots (QDs) directly to a nanowire waveguide. ${ }^{1-6}$ Embedding them in a singlemode nanowire waveguide enhances outcoupling, yet keeps the QD close to the sensing medium thanks to the small dimensions of the nanowire. For this reason, QD-in-NWs are potentially more sensitive to variations in local electric and magnetic fields than QDs. ${ }^{7}$ As a benefit to quantum optics, the QDs maintain their high anti-bunching and narrow emission lines: Combined with the NW waveguide, they make efficient single-photon sources. ${ }^{8}$ In addition, doping of the nanowires can be exploited, for example, to make a single-photon detector by embedding the QDs inside a p-n junction. ${ }^{9-11}$

Several groups have demonstrated the controlled fabrication of vertical nanowires by applying a "top-down" approach using electron-beam lithography (EBL) and reactive ion etching (RIE). ${ }^{12}$ Although this method generates vertical nanowires at specific positions, the surface morphology of these nanowires is damaged by the top-down approach; this damage degrades their optical properties. Additionally, no method can transfer these nanowires to other substrates while preserving their orientation. Two "bottom-up" NW growth approaches - the vapor-liquid-solid (VLS) method, ${ }^{13}$ and the "site-controlled", catalyst-free method by selective area epitaxy ${ }^{14}$ — have been exploited to fabricate QD-in-NWs,. These kinds of nanowires benefit from high-quality surfaces and from the reproducible inclusion of QDs in their matrix. Using the gold-catalyzed VLS method, Borgstom et al. grew GaAs $\mathrm{P}_{1-\mathrm{x}}$ QDs in GaP nanowires. ${ }^{1}$ These high quality QDs, however, had the inconvenient characteristic of extreme polarization anisotropy, ${ }^{15}$ because the NWs had to be transferred to a horizontal orientation for characterization. This orientation-dependence reduces the efficiency of light extraction. Approaches to improve the efficiency of light collection involve photonic design of the shape of the nanowire, including tapering. ${ }^{2}$ A further 
improvement in the QD-in-NW design involved the deposition of a $\mathrm{SiO}_{2}$ layer conformably onto the tapered nanowire. ${ }^{16}$ The top-taper allowed low-loss out-coupling to free space, while the $\mathrm{SiO}_{2}$ thickness allowed further tuning of energy emission of the QD by the application of a homogeneous static strain. These wires could be excited optically head-on.

Heinrich et al. have reported the site-controlled formation of GaAs/AlGaAs QD-in-NWs on EBL-patterned substrates by selective metalorganic chemical vapor deposition (MOCVD). ${ }^{8}$ Although the vertical alignment achieved by this method is optimal for vertical light excitation and emission, the lengths of the nanowires so fabricated were still heterogeneous; the resulting dispersion in lengths limits their use in functional optical devices. Although other methods to control the position and alignment of the QD-in-NWs patterning a substrate by electron-beam have been developed, ${ }^{17}$ the parallel fabrication of vertically oriented QD-in-NWs with uniform predefined lengths remains a challenge. This paper describes an experimental approach which circumvents many of the difficulties of previous methods for preparing QD-in-NWs.

The performance of NWs as optical devices can change (for better or worse) when they are transferred to a new substrate such as a low-index dielectric, a highly-reflective mirror substrate, ${ }^{2,8,18}$ or even an absorbing gold substrate. ${ }^{19}$ Familiar techniques, such as drop-casting or mechanical transfer, allow straightforward transfer of nanowires to the new substrate, but scatter them horizontally and randomly over its surface, and thus complicate further processing (e.g., wet processing or length control) and optical characterization (excitation and collection). ${ }^{20}$ Precise control over the length of the nanowires allows optical cavity effects to be exploited. ${ }^{21}$ Duan et al. controlled the length of CdS nanowires to use them as Fabry-Perot optical cavities, and demonstrated that nanoscale lasers could be made from single semiconductor nanowires. ${ }^{22}$ The generation of highly localized, intense 
monochromatic light inside the constrained geometry of the nanowires requires, besides a well-controlled length of the nanowire, the additional constraint of smooth (and thus reflective) end facets. ${ }^{23} \mathrm{We}$ wished to i) provide a method to facilitate the transfer of QD-inNWs to a reflective substrate (to improve upwards-directed emission), and ii) to control the length of the nanowires, while replacing their terminal catalyst globule with smooth terminal facets to exploit their resonant potential.

During the last decade, our groups have worked on the fabrication of optical devices based on nanowires with implanted active photonic materials. Babinec et al. made singlephoton sources by etching vertical nanowires in a diamond slab implanted with nitrogenvacancy $(\mathrm{NV})$ centers. ${ }^{24}$ Uccelli et al. used a bottom-up approach to fabricate optically active Stranski-Krastanov InAs quantum dots on the facets of GaAs nanowires. Single- and bi-excitons were excited in these high quality QD structures at cryogenic temperatures after the nanowires were transferred from the original GaAs substrate on a Si substrate. ${ }^{25}$ Recently, Heiss et al. developed a versatile QD-in-NW fabrication method that reproducibly selfassembles in core-shell GaAs/AlGaAs nanowires. These quantum dots are highly stable and can be positioned with nanometer precision relative to the nanowire center during the autocatalytic VLS growth process. ${ }^{26}$ This bottom-up approach, in contrast with the top-down diamond system, generates nanowires that systematically contain at least one emitter and makes it possible to generate a variety of complex nanostructures by in-situ doping during the nanowire growth.

In previous work with nanoskiving, Xu et al. fabricated arrays of open and closed-loop nanostructures of gold. ${ }^{27-29}$ These arrays of metallic nanostructures served as mid-IR, frequency-selective surfaces. ${ }^{29}$ The relatively shallow relief of the nanoskived features $(\sim 2 \mu \mathrm{m})$ did not allow a large number of slabs to be cut $(\sim 20)$. Lipomi et al. used 
nanoskiving to pattern the tip of optical fibers with metallic nanostructures, taking advantage of the easy transfer of sectioned epoxy slabs to different substrates. ${ }^{30}$ The use of nanoskiving for the generation of single crystalline, optical nanostructures (such as quantum dots) has not yet been studied.

Here we demonstrate a new fabrication method (which we call "nanoskiving"29) for the fabrication of functional optical devices such as resonators, from a QD-in-NW system. The basic principle of the method is the mechanical nanoscale sectioning of vertically grown GaAs/AlGaAs core-shell nanowires embedded in epoxy. The slabs are obtained using an ultramicrotome to cut in a plane perpendicular to the length of the nanowires. The slabs exhibit a controlled thickness between 80 and $220 \mathrm{~nm}$. This approach allows us to combine precise control of the length of the nanowire achieved by the top-down approach, with the high yield of nanowires generated by bottom-up nanowire growth, by adding only an inexpensive, flexible processing step (nanoskiving) to the bottom-up growth. In addition, the globule of catalyst present at the base of a nanowire to direct its growth is discarded, and nanowires with smooth end-facets can be produced and transferred to a new substrate (for example, a highly-reflective mirror) for use as optical devices.

We chose to nanoskive GaAs/AlGaAs core-shell nanowires with self-assembled AlGaAs quantum dots because they are a high-quality and extremely bright QD-in-NW system. ${ }^{26}$ The structure of the GaAs/AlGaAs core-shell nanowires we sectioned using an ultramicrotome consists of $\mathrm{Al}$-poor $\mathrm{Al}_{\mathrm{x}} \mathrm{Ga}_{1-\mathrm{x}} \mathrm{As}(\mathrm{x} \sim 10 \%)$ quantum dots located between a pure GaAs core and an Al-rich $\mathrm{Al}_{\mathrm{x}} \mathrm{Ga}_{1-\mathrm{x}} \mathrm{As}(\mathrm{x} \sim 75 \%)$ shell. The quantum dots form on the ridge between the core and the shell and are very stable (that is, they survive several months of exposure to air). These QDs exhibit excellent optical properties even when they are located just a few nanometers from the surface: individual quantum dots are very bright (Megahertz 
count rate) and have narrow linewiths (sub-100 $\mu \mathrm{eV}$ ) even without engineering the photonic modes. We chose an Al-rich AlGaAs alloy for the shell, as it is optically transparent in the emission spectrum of the quantum dots.

We used an ultramicrotome (Leica Ultracut UCT) to produce sections of GaAs/AlGaAs core-shell nanowires embedded in sub-100 nm slabs of supporting matrix (“nanoskiving"). ${ }^{29}$ Ultramicrotomes are much more accessible and less expensive than cleanrooms, e-beam writers, and related equipment. Nanoskiving makes it possible, using easy changes in the setting of the ultramicrotome, to control both the length of the nanowires ( $L$, thickness of the sectioned slab) and the angle of the cutting $(\theta)$, and thus to produce nanostructures with very smooth facets. In addition, nanoskiving enable the QD-in-NWs to be manipulated and positioned on arbitrary substrates (such as curved surfaces and over gaps), while retaining the relative in-plane positions of arrays of structures. ${ }^{29}$

The fabrication of QD-in-NW systems using nanoskiving implies significantly different challenges than the fabrication of metallic nanostructures, ${ }^{27,28,30}$ since a precise control on the alignment of the knife of the ultramicrotome and the crystalline planes of the nanowires is required. Figure 1 sketches the nanoskiving process we used to produce sections of GaAs/AlGaAs core-shell quantum-dot-in-nanowires embedded in sub-100 nm slabs of supporting matrix (epoxy) using an ultramicrotome. We grew the GaAs/AlGaAs core-shell nanowires on a silicon wafer, as described elsewhere. ${ }^{26}$ We molded low-surface-energy epoxy (Epo-Tek UVO-114) onto the surface of the wafer (see Fig. S2, S3). The low surface tension of the uncured epoxy allows it to embed the nanowires completely (after degassing to remove air bubbles in a desiccator at 36 Torr for $3 \mathrm{~min}$ ). We then cured the epoxy using UV light $(\lambda=365 \mathrm{~nm})$ and cleaved it from the substrate (see Fig. S4). The epoxy block with embedded nanowires was subsequently sectioned into 80-250 nm-thin slices on an 
ultramicrotome with a diamond blade submerged in water, starting the cutting from the base of the nanowires (the closest to the catalyst particle. See Fig. 1d, S5, and S6). The epoxy slices (with embedded nanowire sections) were transferred to two new substrates, using a "Perfect Loop": ${ }^{29}$ silicon wafers with a 50-nm gold coating, and silicon wafers with a 3- $\mu$ m-thick thermal oxide (see Fig. S7). Finally, the epoxy slab embedding the sections of GaAs/AlGaAs nanowires was removed by etching using an oxygen plasma (200W, 500 mTorr) for 5 minutes, leaving free-standing QD-in-NW slices (see Figure 1f).

Figure 2a shows a scanning electron microscopy (SEM) image of the GaAs/AlGaAs core-shell nanowire forest grown on a silicon wafer using droplets of Ga as catalyst precursors. ${ }^{31}$ After the sectioning process, dark-field microscopy images of the epoxy slabs exhibit multiple bright dots that correspond to the scattering of the light with the GaAs/AlGaAs core-shell nanowire sections embedded in the epoxy (see Figure 2b). Figure $2 \mathrm{c}$ demonstrates that both the vertical orientation, and the sharp hexagonal shape of the nanowires, is preserved after nanoskiving, transferring the slab of epoxy with the nanowire sections to a new substrate, and removing the epoxy matrix of the slab with oxygen plasma.

The self-assembled AlGaAs QDs in GaAs/AlGaAs core-shell nanowires nucleate in the shell grown over the GaAs core. Taking advantage of this effect, it is possible to determine the distance between the QDs and the central axis of the nanowire by modifying the thickness of the GaAs core (by changing the conditions of the nanowire growth). ${ }^{26} \mathrm{GaAs}$ has a smaller bandgap than the AlGaAs QDs; the GaAs core of the nanowires could therefore hinder the optical performance of the QDs by absorbing light in their emission spectrum. It will generally be desirable, for optical applications that require an optimal performance of GaAs/AlGaAs core-shell nanowires, to eliminate the GaAs core after the fabrication of the 
QDs-in-NW systems. The selective etching of the GaAs core directly on the as-grown GaAs/AlGaAs nanowire-structures, however, is difficult to accomplish, since the core is protected by conformal AlGaAs walls, and by the proximity of the substrate. When the nanowires lie horizontally on the substrate (after being transferred by drop-casting, for example) the limited access to the GaAs core also makes it difficult to dissolve the cores of the wires selectively without flushing them off the substrate. Nanoskived wires, in contrast, are firmly positioned on the substrate by the epoxy matrix, and their vertical orientation allows direct access to the GaAs core.

We dissolved the GaAs core of the nanowires by placing a $10-\mu \mathrm{L}$ drop of aqueous citric acid, mixed in a 4:1 volume ratio with hydrogen peroxide (30\%), onto the nanoskived slab. ${ }^{32}$ After $40 \mathrm{~s}$, the substrate was rinsed with deionized water and dried under a stream of nitrogen. This wet etching process completely removed the GaAs cores of the nanowires, thus generating nanoscale "nuts" (see Figure 3). We used an oxygen plasma (200 W, $20 \mathrm{mtorr}, 20 \mathrm{sccm}, 5 \mathrm{~min}$ ) to remove the epoxy matrix, and to leave the vertical nanostructures freestanding over the substrate.

Using nanoskiving to tailor the length of QD-in-NWs also makes it possible to modify the geometry of the GaAs/AlGaAs core-shell nanowires by controlling the cutting angle, $\theta$ (the angle between the plane of the diamond knife of the microtome and the long axis of the nanowire). GaAs/AlGaAs nanowires cleave along the plane (111), when $\theta=0^{\circ}$ (see Figure 3a), or along the plane (110), when $\theta=35^{\circ}$ (see Figure $3 \mathrm{~b}$ ). Modifying the cutting angle requires no more than a straightforward change in settings on the ultramicrotome. After cutting a first slab, all the subsequent slabs will contain nanowires with both ends parallel, and an inclination angle defined by cutting angle $(\theta)$. 
We measured the photoluminescence (PL) of the QD-in-NWs to ensure that the optical properties of the embedded quantum dots were not altered by all the processing steps. The photoluminescence spectra of the QD-in-NWs were collected after the nanoskived slices were transferred to a silicon substrate, and the QDs were located using cryogenic confocal microscopy.

The background photoluminescence of the GaAs core of the nanowire slices makes them appear as bright dots on the confocal image of the nanoskived slab (see Figure 4a). We can identify which of these nanowire slices contain a QD emitter by collecting the PL spectra around the emission frequency of the QDs (660 nm average), see Figure 4b. Nanowire slices without a quantum dot emit light broadly around $825 \mathrm{~nm}$ - the bandgap of GaAs at low temperature $(10 \mathrm{~K})$ - while nanowires with quantum dots embedded exhibit an additional emission peak around $660 \mathrm{~nm}$. The number of QDs per nanowire slice depends on the axial distribution of QDs during growth, which at the same time depends on the thickness and Al content of the nanowire slice. We found that, after nanoskiving, the QD-related PL remains strong while the PL of the GaAs core is strongly diminished despite its larger volume of material; background noise from the GaAs core is thus reduced in favour of signal from the QDs. GaAs luminescence is extremely sensitive to the existence of surface states. The cutting process creates two new non-passivated surfaces on the GaAs, which bends the GaAs bands; this bending prevents radiative recombination of carriers. ${ }^{33}$ At the same time, one would expect the $\mathrm{Al}_{\mathrm{x}} \mathrm{Ga}_{1-\mathrm{x}} \mathrm{As}$ layer to oxidize, and this oxidation to damage the optical properties of the QDs. In practice, we did not observe this kind of damage. This observation suggest that the oxidation remains very superficial.

Figure $4 \mathrm{c}-\mathrm{d}$ demonstrates that the quantum dots embedded into the GaAs/AlGaAs core-shell nanowires remain optically active after the nanoskiving process, and that the 
optical quality of the QDs is conserved. Figure 4c shows a high-excitation-power spectrum (200 $\mu \mathrm{W} / \mu \mathrm{m}^{2}$ laser illumination) of the slice highlighted in Figure $4 \mathrm{~b}$. QDs are populated by more than one exciton at such high excitation power, and, as a result, show a strong broadening of the peaks. Figure $4 d$ shows that the narrow linewidth spectrum of the QDs embedded in the nanowire is recovered when the PL spectrum is collected using a low power laser $\left(2 \mu \mathrm{W} / \mu \mathrm{m}^{2}\right)$. A Lorentzian fit of this peak yields a FWHM around $400 \mu \mathrm{eV}$. Linewidth values down to $100 \mu \mathrm{eV}$ were observed in certain slices, reaching the resolution limit of the spectrometer. These linewidths are slightly higher than the ones reached in as-grown nanowires, most probably due to the sensitivity of the nanowire sections to neighboring interfaces and the associated surface charges. The optical response of the nanowire slices is also very stable in time, even at high power $\left(200 \mu \mathrm{W} / \mu \mathrm{m}^{2}\right)$, and does not show significant blinking, or bleaching of the QD emission peak, over periods of 30 min.

We performed Raman spectroscopy on the QD-in-NW slices to probe the overall quality of the nanowire sections. Figure 5 shows the Raman spectrum corresponding to the single slice whose luminescence spectrum is displayed in Figure 4. Thanks to the vertical orientation of the QD-in-NWs on the substrate, both transverse (TO) and longitudinal (LO) optical phonon modes were excited. The GaAs core holds a LO-mode at $\sim 295 \mathrm{~cm}^{-1}$ clearly visible and a TO-mode at $\sim 270 \mathrm{~cm}^{-1}$ forming the blue shoulder of the TO-related peak. Peaks pertaining to the AlGaAs shell, for first-order Raman scattering, split into two main groups: GaAs-like and AlAs-like. The GaAs-like modes contribute to the broad peak at low frequency, while the AlAs-like are located around $360 \mathrm{~cm}^{-1}$ (TO) and $390 \mathrm{~cm}^{-1}$ (LO). The LO broadening is due to the presence of two peaks; one at low frequency from the $50 \% \mathrm{Al}$ shell and one at higher frequency (prominent due to quasi-resonant conditions) resulting from the Al-rich ( 70\%) planes separating the (110) facet and hosting the QDs. Most interestingly, one can remark the peak at $795 \mathrm{~cm}^{-1}$, which originates from a two-phonon scattering process 
of AlAs-like LOs (2LOs): the presence of 2LOs reveals the quality of the material, as the intensity of second-order processes is usually weak under non-resonant conditions. This shows that the AlGaAs shell material is not fully oxidized, despite several months of storage in air. The overall sharpness of the Raman resonances (coming from both core and shell) and their intensity compared to the silicon substrate demonstrates that the nanowire slices kept their crystalline structure. This validates the nanoskiving concept for applications where sharply defined end-facets are required, as is the case for optical resonators.

We calculated the dependence of the wavelength of the resonant modes with the thickness of the QD-in-NWs using finite-difference time domain (FDTD) simulations (see Fig. 6). Our FDTD simulations show that a portion of the light that reaches the nanowire slice is confined inside the QD-in-NW by total internal reflection. This trapped light travels inside the QD-in-NW in a roughly circular fashion and interferes constructively with itself, forming an optical mode (Fig. 6a). The wavelengths of the optical modes were found by fast Fourier transform (FFT) of the electromagnetic field intensity measured at the center of the QD-in-NW, over the duration of the simulation (discarding the first 100 fs to avoid nonresonant frequencies). Figure $6 \mathrm{~b}$ shows the profile of the optical modes at different resonance frequencies. This resonant modes are all of first order radially, and of order four to seven azimuthally. The quality factor of the optical modes, Q, was calculated by fitting the decay of the total electromagnetic field energy in the simulation volume. We calculated the dependence of the wavelength of the optical modes of a QD-in-NW with thicknesses (see Figure 6c) to demonstrate that it is possible to tune the resonance wavelength of the QD-inNW by changing the thickness of the slice. The thickness of the QD-in-NWs fabricated by nanoskiving can be easily tuned by changing the settings of the ultramicrotome during the cutting process. 
We have demonstrated that nanoskiving provides a versatile method for the fabrication of functional optically-active nanoscale slices based in QD-in-NWs. When sectioning GaAs/AlGaAs core-shell nanowires ( $10 \mu \mathrm{m}$ long), nanoskiving allows the parallel fabrication of a great number of slabs (typically $\sim 120$ ), containing vertically aligned QD-in-NWs with the same length (80-220 nm; the thickness of the nanoskived slab defines the length of the QD-in-NWs). This fabrication method is simple, fast, compatible with different type of QD-in-NWs, and inexpensive when compared with techniques involving high-resolution lithography tools such as EBL or FIB. In addition, the variation of the cutting angle and the cutting thickness during the sectioning process allows for the fabrication of thin QD-in-NWs slices of different shape and aspect ratio, respectively. The epoxy slabs that embed the QD-in-NWs can be transferred to a variety of substrates, while maintaining the vertical orientation of the QD-in-NWs. This uniform orientation makes characterization easier with head-on optical setups, as light is coupled in and out of the vertically aligned wires. The epoxy slab also facilitate post-processing of the nanostructures via wet etching; This slab can, however, also be completely removed by treatment with oxygen plasma prior to the optical characterization of the self-standing QD-in-NWs. We demonstrated this ease of wet-etching by selectively dissolving the optically-absorbing GaAs core with citric acid, but other wet-chemical procedures can also be used. Cryogenic photoluminescence and Raman spectra demonstrate that the quantum dots are functional after sectioning.

The fabrication method described here, at its present level of development, also has two limitations: i) The minimum length of the QD-in-NWs that can be fabricated is limited by the resolution of the ultramicrotome. Thicknesses less than $50 \mathrm{~nm}$ can, in principle be achieved, but are experimentally difficult, and require modifications (e.g. more expensive and fragile diamond knives) of the procedure described here. ${ }^{27,29}$ The length of the QD-in-NWs can, nonetheless, be fabricated over a large range $(50 \mathrm{~nm}-2 \mu \mathrm{m})$, simply by changing the 
thickness of the slab cut by the ultramicrotome. ii) The compression forces applied by the diamond knife cleave the QD-in-NWs only when applied in the same direction as their crystalline plane families (111) and (110); compression forces in other directions, caused by misalignment of the diamond knife, result in shattered nanostructures.

\section{Materials and methods}

\section{Nanowire Growth}

The nanowires were grown by molecular beam epitaxy (MBE) on a DCA P600 system. The cores were grown on a $\mathrm{Si}(111)$ substrate at $640^{\circ} \mathrm{C}$ under a Ga flux equivalent to a planar growth rate of $0.03 \mathrm{~nm} / \mathrm{s}$ and $\mathrm{V} / \mathrm{III}$ flux ratio of 60 , rotating the substrate holder at 7

rpm. ${ }^{34,35}$ To grow the shells, the Ga flux was closed for about $5 \mathrm{~min}$, the arsenic pressure was increased to $2 \times 10^{-6} \mathrm{mbar}$ and the substrate temperature decreased to $460{ }^{\circ} \mathrm{C}$, thus switching the growth direction from axial to radial. ${ }^{36}$ The wires were capped with a $5 \mathrm{~nm}$ GaAs protection layer to avoid oxidation.

\section{Nanoskiving}

Cut-out PDMS molds were placed onto the wafer fragments containing vertical nanowires, and made to seal within the edges of the wafer (see Supporting Information, Fig. S1-S7). Epo-Tek UVO-114 epoxy was poured into the molds, degassed for three minutes in a desiccator (36 Torr), then UV-cured for 30 minutes $(\lambda=365 \mathrm{~nm})$, and unmolded. The epoxy block was cleaved off the wafer with tweezers, then cured another 20 minutes. The epoxy blocks were sliced on a Leica Ultracut UCT microtome into slices $80 \mathrm{~nm}$ to $250 \mathrm{~nm}$ thick, using a diamond knife submerged in water. Slices were transferred within a droplet of water, using a Perfect Loop tool (Diatome, Biel, Switzerland), to two kinds of substrates: silicon wafer supporting a thin evaporated gold film, and silicon wafers with $3 \mu \mathrm{m}$-thick oxide. The substrates were treated with oxygen plasma for two minutes before use, to remove 
absorbed organics and to increase their hydrophilicity. In selected samples, the epoxy was removed by etching by exposure to an oxygen plasma (200W, 500 mTorr) for 5 minutes.

\section{Citric acid etch}

The etchant was prepared by mixing deionized water and citric acid in a 1:1 weight ratio. This solution was then mixed in a $4: 1$ volume ratio with hydrogen peroxide $(30 \%)$. The core of the sectioned nanowires were etched by placing a $50-\mu 1$ drop of etchant onto the epoxy slice with embedded wires. After $30 \mathrm{~s}$ (for a 80-nm thick slice), the substrate was rinsed with deionized water and blown dry with a stream of nitrogen gas. The epoxy slab, which kept the nanowires in place during the etching and rinsing process, was plasma-etched as the final step prior to the optical characterization of the nanostructures.

\section{Scanning Electron Microscopy}

The epoxy cladding was removed from the supported QD-in-NWs, then coated with Pt-Pd in a Cressington HR 208 sputter coater for $60 \mathrm{~s}$ at $40 \mathrm{~mA}$ current. Images were acquired on a Zeiss Ultraplus scanning electron microscope, operating between $4 \mathrm{kV}$ and $5 \mathrm{kV}$, collecting with either the in-lens or Everhart-Thornley secondary electron detector.

\section{Optical Characterization}

The measurements were performed in a cold-finger cryostat at $10 \mathrm{~K}$ (see Fig. S8). The 488-nm line of an argon-krypton laser was used to excite the samples. The laser spot was scanned across the sample in $150 \mathrm{~nm}$ steps. The radiated light was dispersed using an Acton SpectraPro spectrometer with a $1350 \mathrm{l} / \mathrm{mm}$ grating, centered at $830 \mathrm{~nm}$ for the GaAs luminescence and $670 \mathrm{~nm}$ for the QDs luminescence. The low-resolution spectra were acquired on a fiber-coupled Hamamatsu MiniSpec with a 400-1100 nm spectral range (laser power at the entrance of the objective ar. $200 \mu \mathrm{W}$ ). The high resolution spectra were acquired using a 
SpectraPro spectrometer with a 1800 lines per millimeter grating, providing a raw resolution around $100 \mu \mathrm{eV}$ (laser power at the entrance of the objective $\sim 1 \mu \mathrm{W}$ ).

\section{Raman spectroscopy}

The Raman spectrum presented in Fig. 5 was acquired at $10 \mathrm{~K}$ on the very same nanowire slice presented in Fig. 4. The pump was provided by the 488-nm line of an argonkrypton ion laser, close to the $\mathrm{Al}_{60} \mathrm{Ga}_{40} \mathrm{As}$ resonance in order to be as sensitive as possible to alteration done by the skiving process or the subsequent oxidation of the Al-rich shell. The laser light was filtered out by a double grating spectrometer (Acton SpectraPro) in subtractive mode. The inelastically scattered Raman light was dispersed on a $\mathrm{LN}_{2}$-cooled CCD (Princeton Instrument eXcelon) by a third spectrometer (1800 1/mm grating). The pump was linearly polarized and the power set to $0.5 \mathrm{~mW}$ at the entrance of the objective. Integration time was set to $10 \mathrm{~min}$.

\section{FDTD Simulations}

We performed the finite-difference time domain simulations shown in Fig. 6 using commercial FDTD software (Lumerical Solutions). We performed high-resolution 3D simulations using a broadband, linearly polarized, plane-wave source in total field-scattered field (TFSF) mode to remove the effect of the limited simulation size. Perfectly matched layers (PML) served as boundary conditions. A graded mesh was used, designed to discretize accurately the geometries of the QD-in-NW systems. The calculations considered an ideal perfectly hexagonal QD-in-NW, three times the diameter of the physical devices presented earlier, suspended in vacuum. The wires were composed of a $150 \mathrm{~nm}$ diameter GaAs core $(\mathrm{n}=3.83+\mathrm{i} 0.179)$, wrapped in a shell with composition A10.51Ga0.49As $(\mathrm{n}=3.45+$ i 0.002 ) to reach $600 \mathrm{~nm}$ total, then capped in $15 \mathrm{~nm}$-thick GaAs. Total diameter was $630 \mathrm{~nm}$. The simulated QD-in-NW slices were $150 \mathrm{~nm}$ thick. 
Figure 1

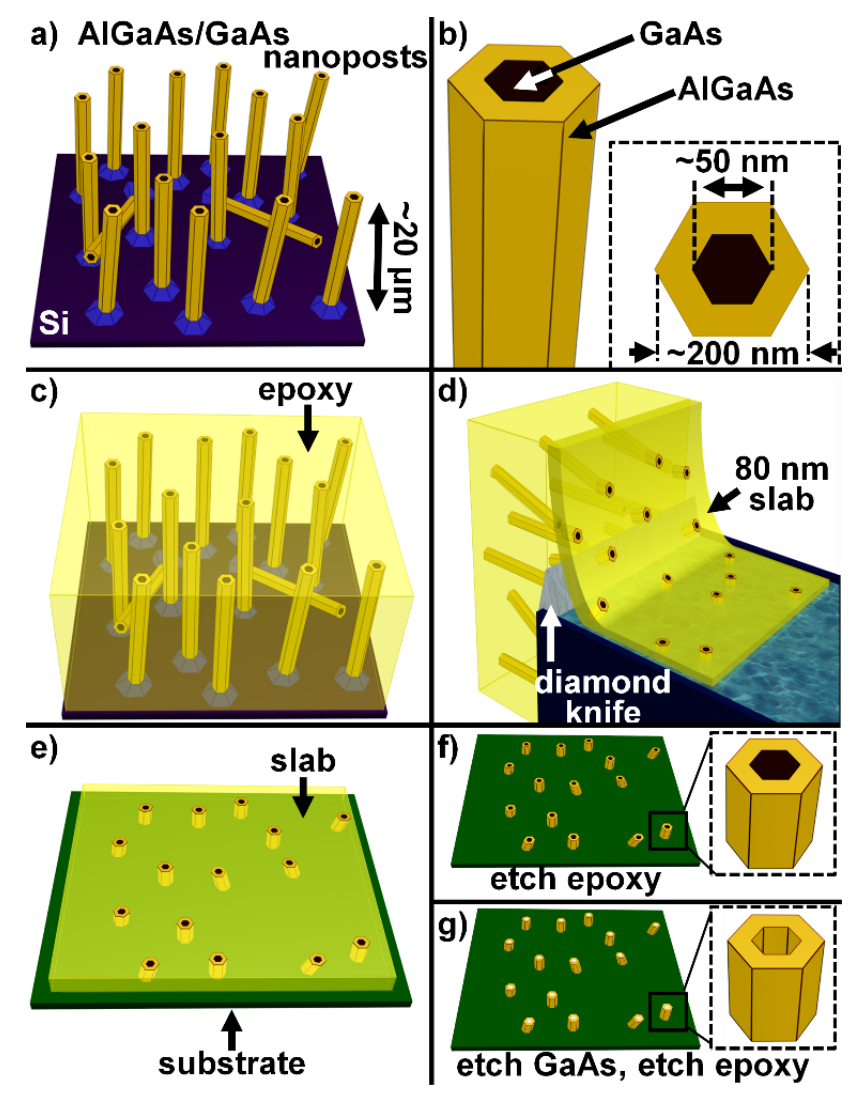

Figure 1. Summary of the nanoskiving process (dimensions stretched for clarity). a) The GaAs/AlGaAs nanowire forest is grown by MBE on a silicon wafer. b) The wires are hexagonal with a GaAs core and $\mathrm{Al} 0.75 \mathrm{Ga} 0.25 \mathrm{As}$ shell. c) The nanowire forest is embedded in epoxy and UV-cured. d) The block of epoxy with the embedded nanowires is then cleaved off and sliced on an ultramicrotome with a diamond blade. e) The slices are transferred on a new substrate. f) Optionally, the epoxy can be etched by oxygen plasma, leaving the slices free-standing on the wafer. g) The core can also be etched by citric acid, leaving hollow-core slices. 
Figure 2
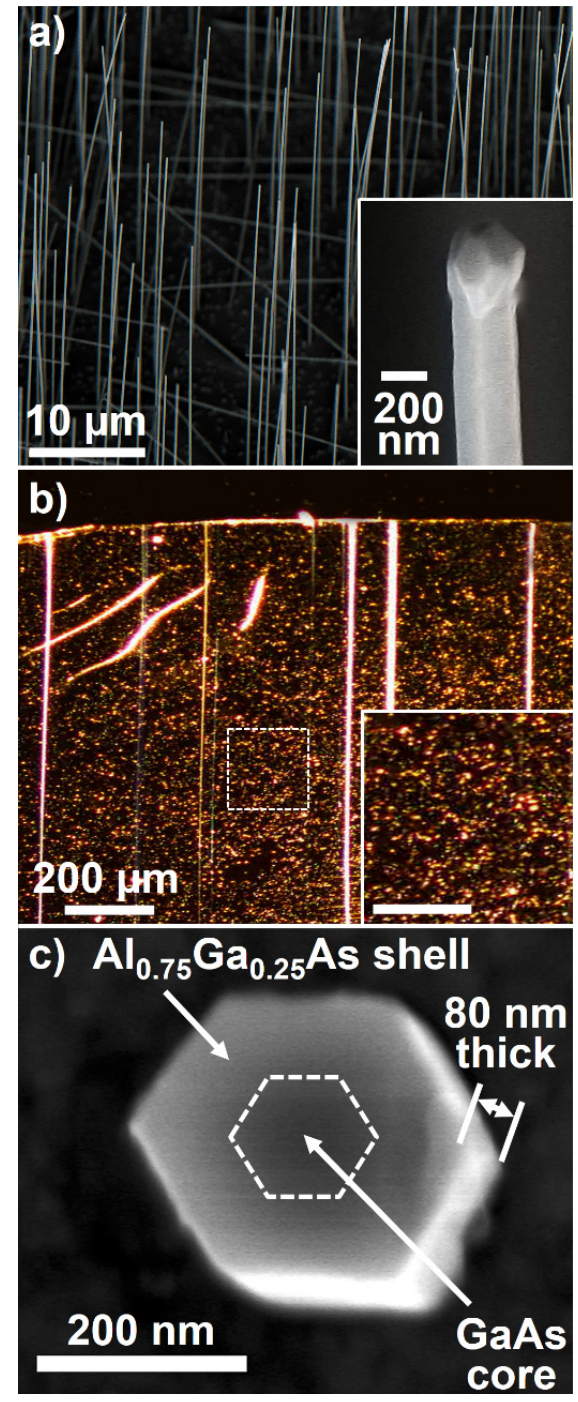

Figure 2. a) Scanning electron microscope (SEM) image of the GaAs/AlGaAs core-shell nanowire forest. The inset shows a high resolution image of one of the tips. b) Dark field optical image of the epoxy slab. The bright dots correspond to the scattering of light by the embedded nanowire sections. The bright vertical lines along the slab correspond to the scattering of light by the defects on the epoxy matrix caused by irregularities in the edge of diamond knife of the ultramicrotome. Oblique bright lines correspond to the scattering of light by the pleats formed on the slab during its transfer to a new substrate. The scale bar of the inset is $100 \mu \mathrm{m}$ long. c) SEM high resolution image of a nanowire section $80 \mathrm{~nm}$ tall. 
Figure 3

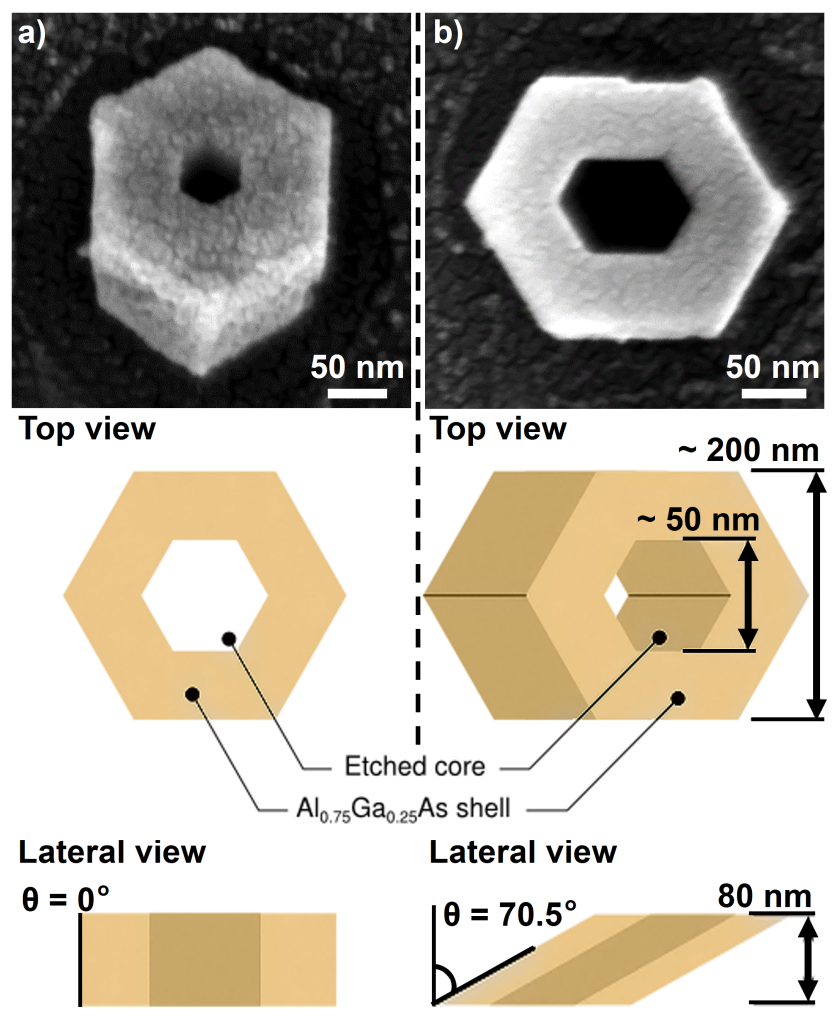

Figure 3. Control of the geometry of the sectioning, and selective etching of the GaAs core of the sectioned GaAs/AlGaAs core-shell nanowires. a) Scanning electron image of an 80-nm-thick slice of a GaAs/AlGaAs nanowire sectioned along its (111) plane after the removal of the GaAs core by citric acid. b) Scanning electron image of a slice of a different GaAs/AlGaAs nanowire (also $80 \mathrm{~nm}$ thick) sectioned along its (110) plane after the selective etching of the GaAs core. SEM images were taken perpendicular to the plane of the substrate. Schemes representing the top and lateral views of the nanostructures illustrate their dimensions. 


\section{Figure 4}
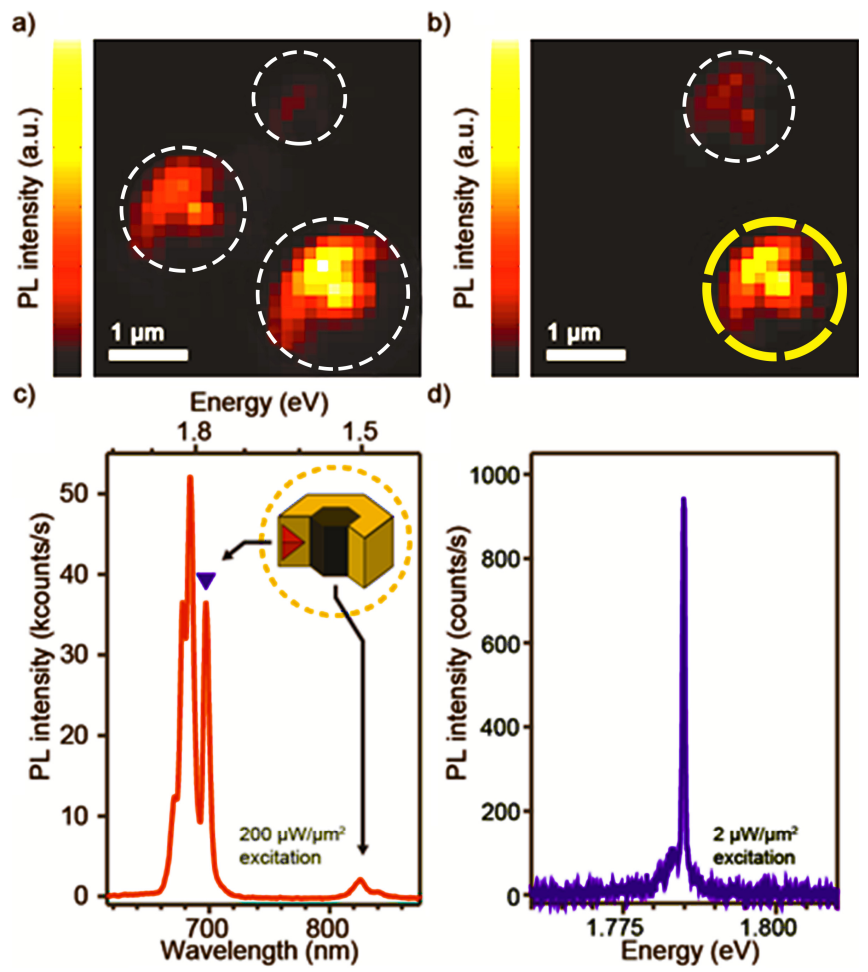

Figure 4. Photoluminescence of nanoskived GaAs/AlGaAs core-shell nanowires. a) Cryogenic confocal scan showing the luminescence of the GaAs core (around $825 \mathrm{~nm}$ ) of three independent nanowire slices (scale bar $1 \mu \mathrm{m}$ ). b) Photoluminescence recorded at $670 \mathrm{~nm}$ in the same area as panel a) reveals that only two of the nanowires contain QDs. c) Full photoluminescence spectrum of the nanoskived QD-in-NW highlighted with a long dashed circle in b). Both the GaAs peak and QD-related peaks can be seen at high excitation power $\left(200 \mu \mathrm{W} / \mu \mathrm{m}^{2}\right)$. d) High resolution spectrum at low excitation power $\left(2 \mu \mathrm{W} / \mu \mathrm{m}^{2}\right)$ reveals a typical narrow single emission peak at $1.7849 \mathrm{eV}(694.6 \mathrm{~nm})$ corresponding to the single exciton recombination in the QD, also marked by the purple arrow in c). 
Figure 5

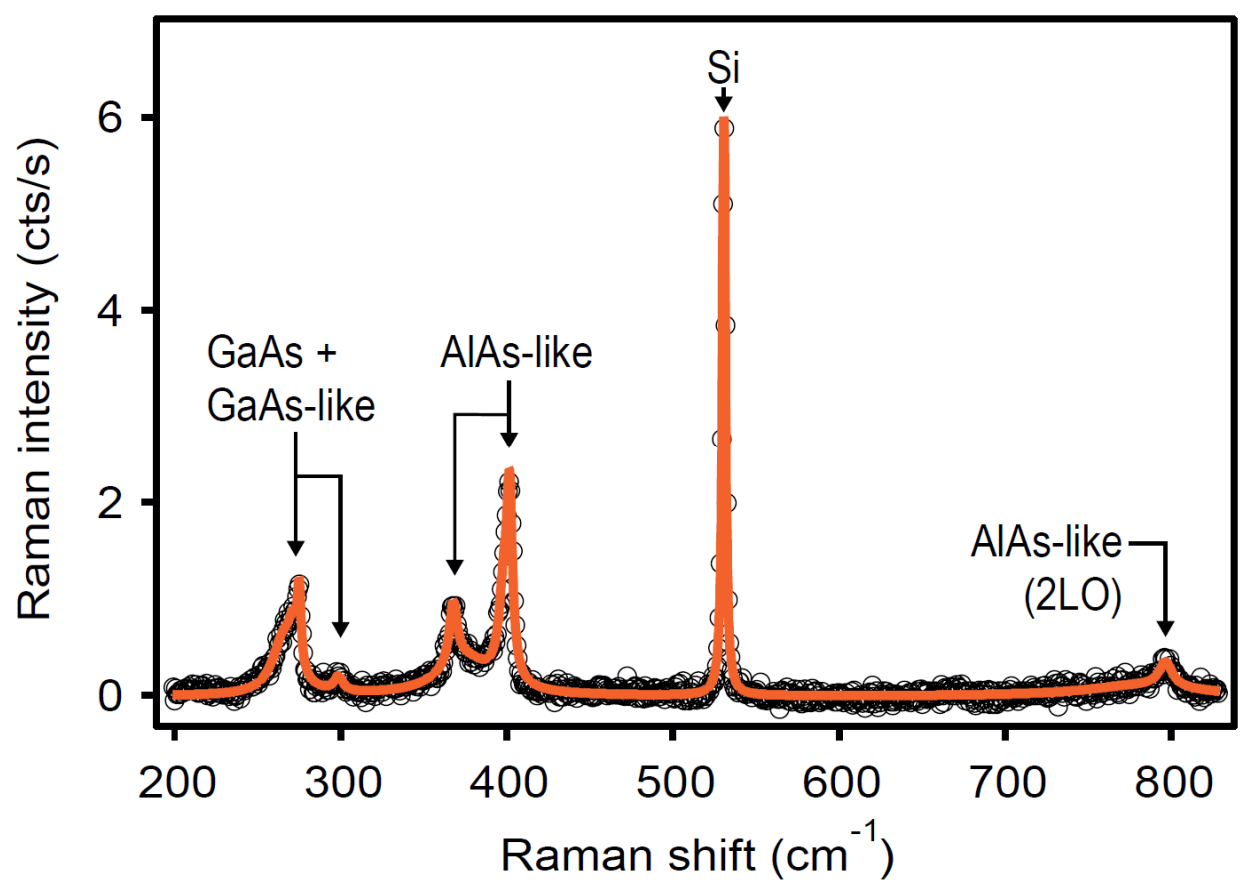

Figure 5. Raman spectrum collected at $10 \mathrm{~K}$ from the NW section measured in Figures 4c-d. The "GaAs" peaks originate from scattering in the GaAs core, whereas the "GaAs-like" and "AlAs-like" originate from the AlGaAs shell. The low-frequency peaks are due to first-order scattering from optical phonons. The highest-frequency peak, located at $800 \mathrm{~cm}^{-1}$, corresponds to two-phonon scattering of the AlAs-like LO phonon branch (shell only). Both the narrow linewidths and high peak intensities show that the material has maintained its quality despite the mechanical stresses it encountered during the nanoskiving process, and after one year of storage under atmospheric conditions. This presence of the 2-LO phonon peak further suggests that the end facets are sharply defined. 
Figure 6

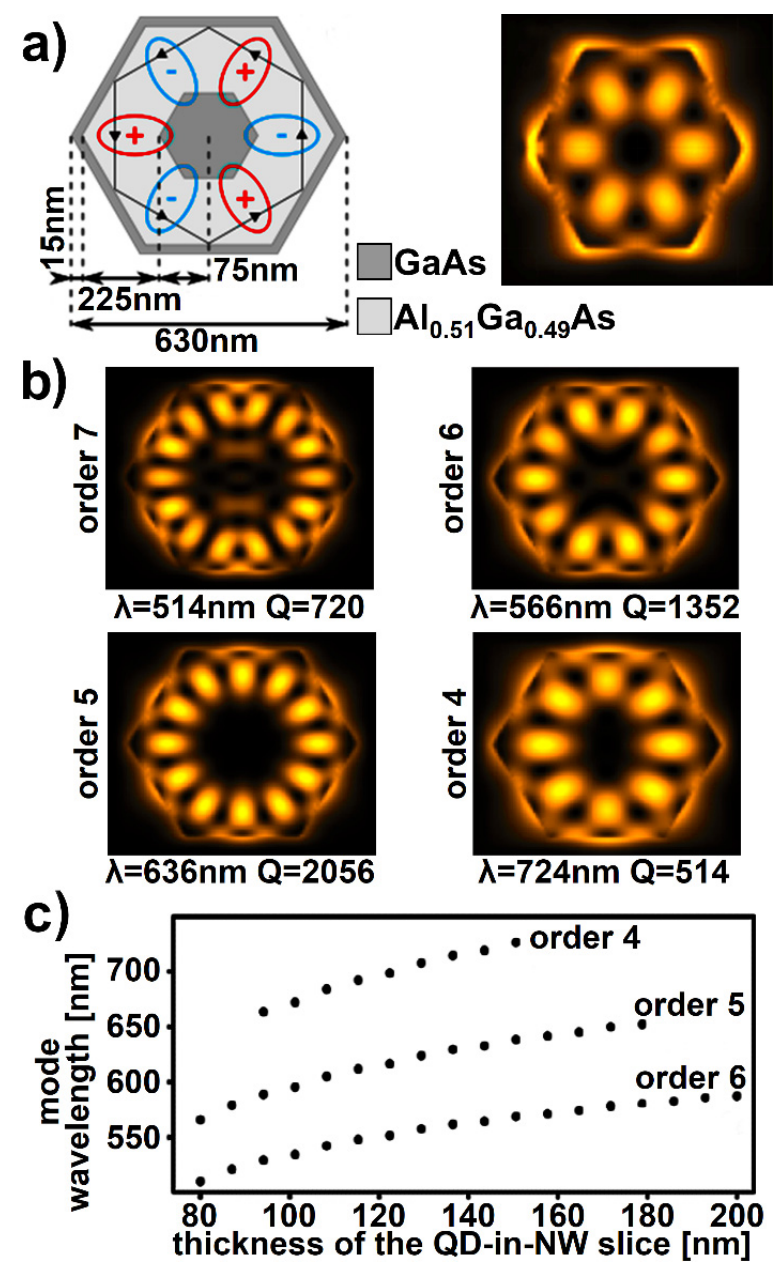

Figure 6. a) Qualitative illustration and FDTD simulation showing how light is trapped inside the QD-in-NW section by total internal reflection. The simulation shows the whispering gallery modes (WGM) formed by the constructive interference of circularly-traveling waves in the 150-nm thick QD-in-NW with no absorption. b) FDTD simulation showing the different resonant modes at various wavelengths with quality factors reaching 2000 c) Thickness dependence of the wavelength of the peak for each WGM. Changing the thickness of the slices of QD-in-NWs with the ultramicrotome tunes the wavelength of the WGMs. 


\section{ASSOCIATED CONTENT}

Supporting Information: More detailed instructions for the nanoskiving process are available in the Supporting Information, along with a schematic of the optical characterization setup. This material is available free of charge via the Internet at http://pubs.acs.org or from the author.

\section{AUTHOR INFORMATION}

\section{Corresponding Authors:}

Prof. George M. Whitesides. Department of Chemistry and Chemical Biology, Harvard University, 12 Oxford Street, Cambridge, MA 02138, USA.

*E-mail: gwhitesides@gmwgroup.harvard.edu

Prof. Marko Lončar. School of Engineering and Applied Sciences, Harvard University, 33 Oxford Street, Cambridge, MA 02138, USA.

*E-mail: loncar@seas.harvard.edu

Author Contributions: E.R-A. and M.H. grew the wires. D.W. and R.V.M. fabricated and imaged the slices. Y.F. characterized the photoluminescence and Raman response of the devices under supervision of A.F.M. D.W performed the FDTD simulations of the nanowires slices. D.W. and R.V.M. wrote the manuscript, through contributions of all authors. G.M.W. and M.K. advised and oversaw the research. All authors have given approval to the final version of the manuscript. $\$$ These authors contributed equally.

\section{Funding Sources:}

Office of Naval Research award N0014-10-1-0942 under subcontract from the University of Pennsylvania.

National Science Fund award ECCS-1028519. 
FP7 People program under the project Marie Curie IOF-275148.

National Science Foundation under NSF Award PHY-0646094.

ERC Starting Grant UpCon.

SNF through DACH program 'Wiring quantum dots'.

SNF through NCCR-QSIT grant number 134506.

Note: The authors declare no competing financial interest.

\title{
ACKNOWLEDGMENTS
}

This research was supported by the National Science Fund award ECCS-1028519. Work focusing on nanoskiving nanowire heterostructures was funded by Office of Naval Research under award N0014-10-1-0942 under subcontract from the University of Pennsylvania. R.V.M. acknowledges funding by the FP7 People program under the project Marie Curie IOF-275148. This work was performed in part using the facilities of the Center for Nanoscale Systems (CNS), a member of the National Nanotechnology Infrastructure Network (NNIN), which is supported by the National Science Foundation under NSF Award PHY-0646094. CNS is part of the Faculty of Arts and Sciences at Harvard University. AFiM thanks support from ERC Starting Grant 'UpCon'. AFiM and YF thank funding from SNF through NCCR-QSIT and DACH 'Wiring Quantum Dots'.

\author{
ABBREVIATIONS \\ QD, Quantum Dot; NW, Nanowire; QD-in-NW, Quantum Dot in Nanowire; PL, \\ Photoluminescence; EBL, Electron Beam Lithography; VLS, Vapor-Liquid-Solid; CVD, \\ Chemical Vapor Deposition; MOCVD, Metalorganic Chemical Vapor Deposition; NV, \\ Nitrogen-Vacancy; RIE, Reactive Ion Etching; MBE, Molecular Beam Epitaxy; PECVD, \\ Plasma-Enhanced Chemical Vapour Deposition.
}




\section{REFERENCES}

1. Borgström, M. T.; Zwiller, V.; Müller, E.; Imamoglu, A. Nano Lett. 2005, 5, 1439-1443.

2. Claudon, J.; Bleuse, J.; Malik, N. S.; Bazin, M.; Jaffrennou, P.; Gregersen, N.; Sauvan, C.; Lalanne, P.; Gérard, J.-M. Nat. Photonics 2010, 4, 174-177.

3. Bleuse, J.; Claudon, J.; Creasey, M.; Malik, N. S.; Gèrard, J.-M.; Maksymov, I.;

Hugonin, J.-P.; Lalanne, P. Phys. Rev. Lett. 2011, 106, 103601.

4. Bulgarini, G.; Reimer, M. E.; Zehender, T.; Hocevar, M.; Bakkers, E. P.; Kouwenhoven, L. P.; Zwiller, V. Appl. Phys. Lett. 2012, 100, 121106.

5. Krogstrup, P.; Jorgensen, H. I.; Heiss, M.; Demichel, O.; Holm, J. V.; Aagesen, M.; Nygard, J.; Fontcuberta i Morral, A. Nat. Photonics 2013, 7, 306-310.

6. Wallentin, J.; Anttu, N.; Asoli, D.; Huffman, M.; Åberg, I.; Magnusson, M. H.; Siefer, G.; Fuss-Kailuweit, P.; Dimroth, F.; Witzigmann, B. Science 2013, 339, 1057-1060.

7. Kouwen, M. P. v.; Reimer, M. E.; Hidma, A. W.; van Weert, M. H.; Algra, R. E.;

Bakkers, E. P.; Kouwenhoven, L. P.; Zwiller, V. Nano Lett. 2010, 10, 1817-1822.

8. Heinrich, J.; Huggenberger, A.; Heindel, T.; Reitzenstein, S.; Höfling, S.; Worschech, L.; Forchel, A. Appl. Phys. Lett. 2010, 96, 211117.

9. Bulgarini, G.; Reimer, M. E.; Hocevar, M.; Bakkers, E. P.; Kouwenhoven, L. P.; Zwiller, V. Nat. Photonics 2012, 6, 455-458.

10. Minot, E. D.; Kelkensberg, F.; Van Kouwen, M.; Van Dam, J. A.; Kouwenhoven, L. P.; Zwiller, V.; Borgström, M. T.; Wunnicke, O.; Verheijen, M. A.; Bakkers, E. P. Nano Lett. 2007, 7, 367-371.

11. Colombo, C.; Heiss, M.; Gratzel, M.; Fontcuberta i Morral, A. Appl. Phys. Lett. 2009, 94, 173108.

12. Hobbs, R. G.; Petkov, N.; Holmes, J. D. Chem. Mater. 2012, 24, 1975-1991.

13. Wagner, R.; Ellis, W. Appl. Phys. Lett. 1964, 4, 89-90. 
14. Motohisa, J.; Noborisaka, J.; Takeda, J.; Inari, M.; Fukui, T. J. Cryst. Growth 2004, 272, 180-185.

15. Wang, J.; Gudiksen, M. S.; Duan, X.; Cui, Y.; Lieber, C. M. Science 2001, 293, $1455-$ 1457.

16. Bouwes Bavinck, M.; Zieliński, M.; Witek, B. J.; Zehender, T.; Bakkers, E. P.; Zwiller, V. Nano Lett. 2012, 12, 6206-6211.

17. Tatebayashi, J.; Ota, Y.; Ishida, S.; Nishioka, M.; Iwamoto, S.; Arakawa, Y. Appl. Phys. Lett. 2012, 100, 263101.

18. Heiss, M.; Fontcuberta i Morral, A. Appl. Phys. Lett. 2011, 99, 263102.

19. Xu, H.; Wright, J. B.; Hurtado, A.; Li, Q.; Luk, T.-S.; Figiel, J. J.; Cross, K.; Balakrishnan, G.; Lester, L. F.; Brener, I. Appl. Phys. Lett. 2012, 101, 221114.

20. Pauzauskie, P. J.; Yang, P. Mater. Today 2006, 9, 36-45.

21. Li, Q.; Wright, J. B.; Chow, W. W.; Luk, T. S.; Brener, I.; Lester, L. F.; Wang, G. T. Opt. Express 2012, 20, 17873-17879.

22. Duan, X.; Huang, Y.; Agarwal, R.; Lieber, C. M. Nature 2003, 421, 241-245.

23. Zimmler, M. A.; Capasso, F.; Müller, S.; Ronning, C. Semicond. Sci. Technol. 2010, 25, 024001.

24. Babinec, T. M.; Hausmann, B. J.; Khan, M.; Zhang, Y.; Maze, J. R.; Hemmer, P. R.; Lončar, M. Nat. Nanotechnol. 2010, 5, 195-199.

25. Uccelli, E.; Arbiol, J.; Morante, J. R.; Fontcuberta i Morral, A. ACS Nano 2010, 4, 59855993.

26. Heiss, M.; Fontana, Y.; Gustafsson, A.; Wüst, G.; Magen, C.; O’Regan, D.; Luo, J.; Ketterer, B.; Conesa-Boj, S.; Kuhlmann, A.; Houel, J.; Russo-Averchi, E.; Morante, J.; Cantoni, M.; Marzari, N.; Arbiol, J.; Zunger, A.; Warburton, R.; Fontcuberta i Morral, A. Nat. Mater. 2013, 12, 439-444. 
27. Xu, Q.; Bao, J.; Rioux, R. M.; Perez-Castillejos, R.; Capasso, F.; Whitesides, G. M. Nano Lett. 2007, 7, 2800-2805.

28. Lipomi, D. J.; Kats, M. A.; Kim, P.; Kang, S. H.; Aizenberg, J.; Capasso, F.; Whitesides, G. M. ACS Nano 2010, 4, 4017-4026.

29. Lipomi, D. J.; Martinez, R. V.; Whitesides, G. M. Angew. Chem., Int. Ed. 2011, 50, $8566-8583$.

30. Lipomi, D. J.; Martinez, R. V.; Kats, M. A.; Kang, S. H.; Kim, P.; Aizenberg, J.; Capasso, F.; Whitesides, G. M. Nano Lett. 2011, 11, 632-636.

31. Colombo, C.; Spirkoska, D.; Frimmer, M.; Abstreiter, G.; Fontcuberta i Morral, A. Phys. Rev. B 2008, 77, 155326.

32. Kim, J.-H.; Lim, D. H.; Yang, G. M. J. Vac. Sci. Technol., B 1998, 16, 558-560.

33. Demichel, O.; Heiss, M.; Bleuse, J.; Mariette, H.; Fontcuberta i Morral, A. Appl. Phys. Lett. 2010, 97, 201907.

34. Uccelli, E.; Arbiol, J.; Magen, C.; Krogstrup, P.; Russo-Averchi, E.; Heiss, M.; Mugny, G.; Morier-Genoud, F. o.; Nygård, J.; Morante, J. R. Nano Lett. 2011, 11, 3827-3832.

35. Rüffer, D.; Huber, R.; Berberich, P.; Albert, S.; Russo-Averchi, E.; Heiss, M.; Arbiol, J.; Fontcuberta i Morral, A.; Grundler, D. Nanoscale 2012, 4, 4989-4995.

36. Heigoldt, M.; Arbiol, J.; Spirkoska, D.; Rebled, J. M.; Conesa-Boj, S.; Abstreiter, G.; Peiró, F.; Morante, J. R.; Fontcuberta i Morral, A. J. Mater. Chem. 2009, 19, 840-848. 
For TOC only

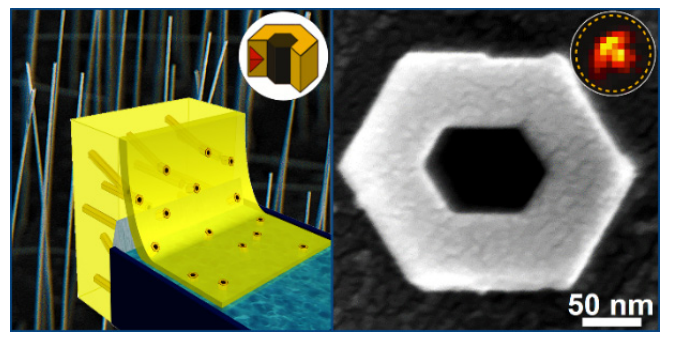

